\title{
Reprojection Flow for Image Registration Across Seasons
}

Shane Griffith
sgriffith7@ @gatech.edu

Cédric Pradalier ${ }^{2,3}$

cedric.pradalier@georgiatech-metz.fr

\author{
${ }^{1}$ College of Computing, \\ Georgia Institute of Technology, \\ Atlanta, USA \\ ${ }^{2}$ GeorgiaTech Lorraine, \\ Metz, France \\ ${ }^{3}$ CNRS UMI 2958 GT-CNRS, \\ Metz, France
}

We address the problem of robust visual data association across seasons and viewpoints. The predominant methods in this area are typically appearance-based, which lose representational power in outdoor and natural environments that have significant variation in appearance. After a natural environment is surveyed multiple times, we recover its $3 \mathrm{D}$ structure in a map, which provides the basis for robust data association. Our approach is called Reprojection Flow (see Fig. 1).

A map can make robust data association possible, but acquiring one that is composed of landmarks from different seasons is a feat in and of itself. First, images are registered (low-res) between near-time surveys to identify images of the same scenes (aided by GPS). Full resolution image registration is performed on the set that aligns well in order to acquire inter-survey observations of KLT-tracked landmarks. A map is recovered from the set of intra- and inter-survey landmark observations using visual SLAM.

Given the optimized map and camera
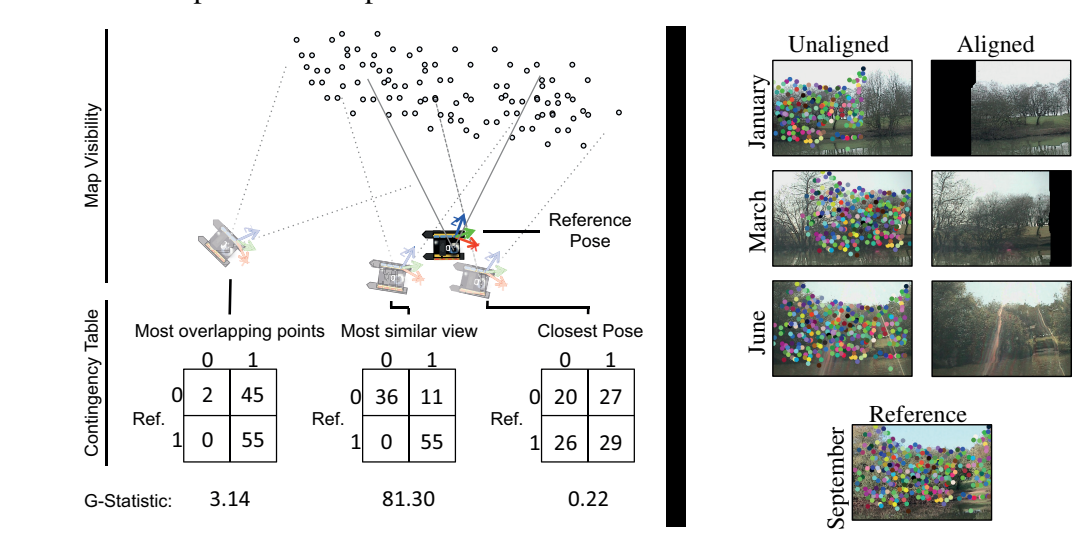

poses, reprojected map points are used for 1) appearance-invariant viewpoint selection and 2) the robust registration of images. First, images of the same scenes from multiple surveys are found by maximizing the co-visibility of reprojected map points. Second, the pixel locations of reprojected map points are used to indicate correspondences between them. This reprojection flow directly provides sparse data association among images of the same scenes, which is applied with matching constraints to maximize the use of appearance-invariant information.

We evaluated this approach using a dataset of 24 surveys of a natural environment that span over a year. This approach significantly improves dense correspondence across seasons compared to SIFT Flow [1]. It also provides robustness to changes in viewpoint.

[1] Ce Liu, Jenny Yuen, and Antonio Torralba. SIFT Flow: Dense correspondence across scenes and its applications. PAMI, 33(5):978-994, 2011.

Figure 1: Reprojection Flow: left) Finding the most similar view by maximizing co-visibility using the Gstatistic, compared to a closest pose heuristic, and a heuristic to maximize the number of overlapping points. The contingency tables are shown for each case. right) Using reprojected map points to guide image registration. The KLT points of the reference image are shown projected onto the unaligned images. 